\title{
Nanohydroxyapatite Silicate-Based Cement Improves the Primary Stability of Dental Implants: An In Vitro Study
}

\author{
Hooman Khorshidi, ${ }^{1}$ Saeed Raoofi,, Maryam Najafi, ${ }^{1}$ \\ Mohammad Hassan Kalantari, ${ }^{2}$ Jamshid Khorshidi Malahmadi, ${ }^{3}$ and Reza Derafshi ${ }^{2}$ \\ ${ }^{1}$ School of Dentistry, Shiraz University of Medical Sciences, Shiraz, Iran \\ ${ }^{2}$ Department of prosthodontics, School of Dentistry, Shiraz University of Medical Sciences, Shiraz, Iran \\ ${ }^{3}$ Department of Mechanical Engineering, Hormozgan University, Bandar Abbas, Iran \\ Correspondence should be addressed to Saeed Raoofi; raoofis@sums.ac.ir
}

Received 3 November 2016; Revised 15 December 2016; Accepted 15 January 2017; Published 19 February 2017

Academic Editor: Michele Iafisco

Copyright (C) 2017 Hooman Khorshidi et al. This is an open access article distributed under the Creative Commons Attribution License, which permits unrestricted use, distribution, and reproduction in any medium, provided the original work is properly cited.

\begin{abstract}
Objectives. Insufficient cortical bone volume when placing implants can lead to lack of primary stability. The use of cement as a bone fill material in bone defects around dental implant could result in better clinical outcome. HA has shown excellent biological properties in implant dentistry. The purpose of this study was to evaluate the effect of nanohydroxyapatite powder (Nano-HA) in combination with accelerated Portland cement (APC) on implant primary stability in surgically created circumferential bone defects in a bovine rib in vitro model. Materials and Methods. Sixteen bovine rib bones and thirty-six implants of same type and size $(4 \mathrm{~mm} \times 10 \mathrm{~mm})$ were used. Implants were divided into six groups: no circumferential bone defect, defect and no grafting, bone chips grafting, Nano-HA grafting, APC grafting, and Nano-HA mixed to APC grafting (Nano-HA-APC). Circumferential defects around the implants were prepared. The implant stability quotient (ISQ) values were measured before and after the grafting. Results. APC exhibited the highest ISQ values. A significant increase of ISQ values following the grafting of Nano-HA-APC (18.08 \pm 5.82$)$ and APC alone (9.50 \pm 4.12$)$ was achieved. Increase of ISQ values after 72 hours was $24.16 \pm 5.01$ and $17.58 \pm 4.89$, respectively. NanoHA grafting alone exhibited the least rise in ISQ values. Conclusions. Nanohydroxyapatite silicate-based cement could improve the primary stability of dental implants in circumferential bone defect around implants.
\end{abstract}

\section{Introduction}

Primary stability is defined as the degree of the mechanical anchoring established when placing an implant. High primary stability is critical for a successful osseointegration of dental implants [1]. A safe primary stability is positively attendant with a secondary stability (osseointegration) [2]. Weakened primary implant stability has been demonstrated to threaten the osseointegration process [3]. Several variations in surgical technique have been described to assist in enhancing primary stability of dental implants, in order to increase the success rate of dental implants and to reduce the overall treatment duration $[1,4,5]$. Insufficient cortical bone volume when placing implants can lead to lack of primary stability. Using a bone graft material around the arranged implant beds was also suggested as a technique to overcome insufficient primary stability of the dental implant [6]. Some materials were assessed for this purpose and they demonstrated encouraging results $[6,7]$. Calcium-silicate cements, like MTA (mineral trioxide aggregate) and other Portlandderived materials, are hydraulic cements mainly composed of tricalcium and dicalcium silicate, tricalcium aluminate, and calcium sulphate. A longlist of new materials based on the original Portland cement formulation and/or with minor modifications has been introduced in dental practice [8]. The setting time of Portland cement can be decreased by adding calcium chloride $\left(\mathrm{CaCl}_{2}\right)$, known as accelerated Portland cement (APC) [9]. Nevertheless, a generic name for this class of materials, Hydraulic Silicate Cement (HSC), has been proposed [10]. It has been suggested that HSC materials can possess the properties to nucleate apatite and may play a positive role as a cell differentiation promoter and also for 
stimulating tissue repair, osteogenesis, and cementogenesis [8]. In a study on "Kinetics of apatite formation in SBF of a HCSC Portland-based cement monitored over time by SEM and EDX analyses," it has been reported that after 7-day soaking in SBF the surface became covered by aggregated spherulites and consequently with long (28 days) soaking times the cement surface shifted from a calcium silicate to a calcium phosphate composition [8]. Kinetic reaction is directly affected by the high surface area of nanoparticles. A number of calcium phosphates (including hydroxyapatite) were introduced in experimental HCS to serve as a reservoir of phosphate and apatite deposits $[8,11]$. APC has the advantage of good handling and manipulation compared other HSC $[9,12]$. Easy handling of bone substitutes in implant dentistry is crucial. For many clinical applications, the main limitation of HA granules in implant surgery was the difficult handling. Nevertheless, hydroxyapatite is a wellknown bone substitute [13]. Hydroxyapatite shows excellent biocompatibility with soft tissues, making it an ideal candidate for dental implants [14]. However, it is not expected that excellent biological properties of HA can increase the early fixation of implants. Early fixation (or in other words primary stability) understood as the mechanical anchoring right after placing an implant. Recently, considerable researches have been carried out on the nanohydroxyapatite [15-18]. HA particles with nanometer sizes $(<100 \mathrm{~nm})$ are favorable for use as implant donor substances, because they simulate the nanostructure of the inorganic phase in natural bones [15]. On the other hand, HA nanoparticles were found to provide a high surface area per HA volume, resulting in increased cellular adherence, proliferation, and osteoblast adhesion [15, 18]. Therefore, the aim of this study was to evaluate the effect of nanohydroxyapatite powder (Nano-HA) in combination with APC on implant primary stability in surgically created circumferential bone defects in a bovine rib in vitro model.

\section{Materials and Methods}

Sixteen frozen bovine rib bones from an Iranian bull or cow were utilized in this study. The rib bones were left at room temperature for $2 \mathrm{~h}$; they were stabilized for the drilling procedure, grafting, and measurements, and their periosteum was removed before drilling. 36 dental implants with an $11^{\circ}$ Morse taper, internal connection-type, and with a self-tapping fixture design were used (KME Co, Iran). All implants have the same size $(4 \mathrm{~mm} * 10 \mathrm{~mm})$.

A pilot study was conducted to determine the depth of defect that affects the primary stability of the implant. The drilling sequence was performed according to the manufacturer's instructions and implant beds were prepared for an implant of $3.5 \mathrm{~mm}$ in diameter and $10 \mathrm{~mm}$ in length. Subsequently, circumferential cortical bone defects were prepared by perforating a defect with a diameter of $6.5 \mathrm{~mm}$. The depth of the defect gradually was increased and the ISQ value measured. By the depth of $3 \mathrm{~mm}$, a sudden reduction in the ISQ value (decrease of 15-25 units) was observed; thus, it was decided to create a circumferential defect by the depth of $3 \mathrm{~mm}$ and a diameter of $6.5 \mathrm{~mm}$ around each implant. Moreover, the cross-section of the rib was prepared to find

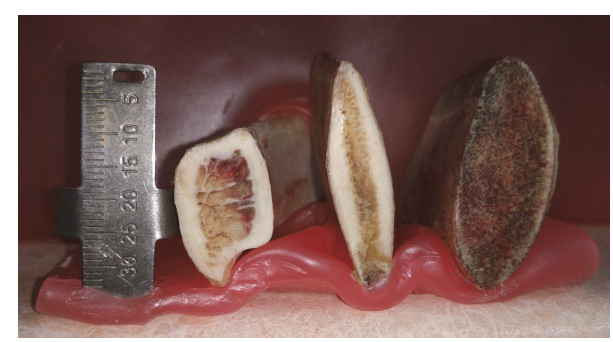

FIGURE 1: Bone section of different parts of rib. The beginning part of the ribs was used in this study (adjacent to the index in the picture).

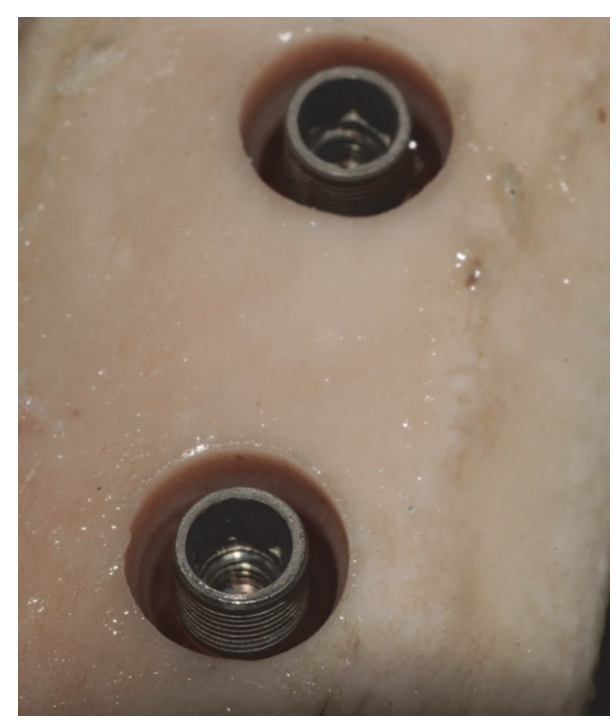

FIgURE 2: Preparation of circumferential bone defect and implant insertion.

the appropriate portion with the best volume and density of the available bone. It was decided to use the beginning part of dorsal surface of all ribs (near the head of the rib that is connected to the spinal cord), in this experiment (Figure 1).

In each rib bone, 2-3 implant beds were prepared in the mentioned area, and the centers of the implant beds were $40 \mathrm{~mm}$ apart. There was 6 groups in the experiment: (1) positive control group: no defect, (2) negative control group: defect and no grafting, (3) defect and bone chips grafting, (4) defect and Nano-HA grafting, (5) defect and APC grafting, and (6) defect and Nano-HA-APC grafting. For each group, 6 implants were utilized. The drilling sequence was performed according to the manufacturer's instruction and implant beds were prepared by $3.5 \mathrm{~mm}$ diameter and $10 \mathrm{~mm}$ length. Subsequently, for creating the circumferential defect around implant, drilling continued with a shorter $8 \mathrm{~mm}$ drill with $6.5 \mathrm{~mm}$ diameter. Thus, after installation of implant there was $1.5 \mathrm{~mm}$ circumferential defect around the implant with $8 \mathrm{~mm}$ depth (Figure 2).

Bone chips were collected during drilling. However, it was an autogenous bone and thus do not require any further preparation. Nano-Ha (Pardis Pajoohesh Fanavaran Yazd, Yazd Science and Technology Park, Yazd, Iran) did not require any preparation as well. 
To prepare APC, $10 \%$ vol calcium chloride (Kimia Material Co, Iran) was added to $90 \%$ vol of Portland cement type 1 (Fars Cement Co, Iran). Since manipulation and handling of the material is a matter of major importance, therefore several combinations of HA and APC by volume were evaluated and the mixture of $75 \%$ vol of Nano-HA and 25\% vol of APC was elected. In addition a pilot study was done for evaluating the material and the specimens $(n=8)$ were fabricated according to standards for the compressive and tensile strength test by test machine Zwick/Roell Z020. The APC and Nano-HA-APC cements were placed in cylindrical molds with $15 \mathrm{~mm}$ length and $10 \mathrm{~mm}$ in diameter. They were stored at room temperature for $48 \mathrm{~h}$, and then the samples were removed from the mold. The compressive and tensile strength tests were performed in test machine with $1 \mathrm{~N}$ preload, at $0.1 \mathrm{~mm} /$ minute speed. The maximum stress was recorded in $\mathrm{MPa}$, using the maximum compressive and tensile force as well as the diameter of the cylinders ( $\mathrm{MPa}$ $=\mathrm{N} / \mathrm{mm}^{2}$ ). Compressive and tensile strength of APC were measured as 1.18 (one tested specimen) and $0.72 \mathrm{MPa}$ (one tested specimen). Compressive and tensile strength of NanoHA-APC were measured as 1.27 (one tested specimen) and $0.54 \mathrm{MPa}$ (two tested specimens, SD: 0.18 MPa). Considering that only one or two samples were tested in this pilot study, it is difficult to conclude that mixing APC with Nano-HA would increase compressive strength and/or reduce tensile strength compared to APC.

2.1. Implant Insertion, Grafting, and ISQ Measurement. Resonance Frequency Analysis (RFA) measures the frequency with which a device vibrates. The stability of a dental implant can be determined as the resonance frequency changes with different stabilities. The implant stability quotient (ISQ) is a global standard for measuring implant stability as measured by RFA. ISQ scale ranges from 1 to 100, but the acceptable stability range lies between 55 and 85 . Implants were placed in each prepared hole and ISQ values were measured four times from the four perpendicular directions with an Osstell instrument and smart peg no. 47. APC was mixed with water at a $2: 1$ ratio $[9,12]$; Nano-HA-APC was mixed with water at a 3:1 ratio (with consult by a biomaterial master) [19]. Graft materials were inserted into defect by a spatula. ISQ values were measured four times from the four perpendicular same directions again. APC and Nano-HA-APC were allowed to harden for $20 \mathrm{~min}$ before second measurement. Lastly, the ISQ values will be measured again after $72 \mathrm{~h}$, after APC and Nano-HA-APC grafting, because these two materials had setting (Figure 3).

ISQ value was measured in four directions for each implant before and after grafting and then compared ISQ value changes before and after grafting in the same direction and same implant. ISQ values were measured before grafting (ISQ 1), after grafting (ISQ 2), and after $72 \mathrm{~h}$ (ISQ 3) in the same directions and same implant.

2.2. Statistical Analysis. All quantitative data were expressed as the mean and standard deviation. Statistical analysis was performed through Kolmogorov-Smirnov $t$-test, nonparametric Kruskal-Wallis, Mann-Whitney $U$ tests, and

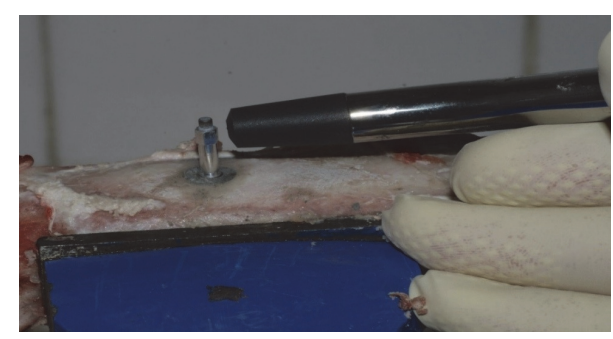

FIGURE 3: Insulation of smart peg on implant and measurement of ISQ value by Osstell device.

Wilcoxon test using SPSS version 22 (SPSS Ltd., Hong Kong). A value of $P<0.05$ was considered statistically significant.

\section{Results and Discussion}

ISQ value for bone without any defect was measured by four different directions in 6 implants. The maximum ISQ value was 76 and the minimum ISQ value was 68 . The mean ISQ value for intact bone was 72.25. The changes in ISQ value before and after the application of each graft are shown in Table 1 .

The mean increase of ISQ values after grafting was statistically significant for every grafting material except for Nano-HA grafting group ( $P=0.000$ for APC and Nano-HAAPC, $P=0.001$ for bone chips, $P=0.564$ for Nano-HA).

While increase in ISQ values after APC grafting was compared with Nano-HA-APC grafting, the increase in ISQ values was not significant $(P=0.097)$. The ISQ improvement in APC and Nano-HA-APC groups in the first 24 hrs was 18.08 and 9.5, respectively. But increasing of ISQ value in the period of $24-72 \mathrm{hrs}$ was dissimilar in the groups. (The difference between ISQ 2 and ISQ 3 was 6 for APC and 8 for Nano-HA-APC.) It seems that this difference would be related to incorporation of nanoparticles in the Nano-HAAPC cement.

In this study, the application of Nano-HA in combination with APC (Nano-HA-APC) around dental implants with insufficient primary stability was assessed as bone cement grafting. This study was not intended to explore Nano-HA instead of APC. The object of this study was to explore the potential mechanical advantages of APC when added to the Nano-HA as a bone graft substitute in clinical implant surgeries. One of these clinical implications would be providing the primary implant stability in circumferential bone defects around the implants. Results of the current study indicated a significant improvement in primary stability following the grafting of APC, Nano-HA-APC, and bone chips in all implants compared to Nano-HA (Figure 4).

This improvement of primary stability was greater by grafting APC and Nano-HA-APC. By grafting of APC, improvement similar to intact bone and even superior to intact bone was achieved. On the other hand, the increase of primary stability by bone chips was not as much as APC and Nano-HA-APC. Nano-HA grafting did not affect the low primary stability. These results are in line with the results 
TABLE 1: Comparison of effect of different graft material on ISQ value of dental implants.

\begin{tabular}{lccccc}
\hline & APC & Nano-HA and APC & Nano-HA & Bone chips & No grafting \\
\hline ISQ 1 & $50.75 \pm 4.05$ & $42.33 \pm 6.45$ & $46.58 \pm 6.95$ & $41.96 \pm 8.04$ & $48.21 \pm 8.30$ \\
ISQ 2 & $68.83 \pm 4.50$ & $51.83 \pm 5.07$ & $46.71 \pm 6.65$ & $47.50 \pm 6.17$ & $48.21 \pm 8.30$ \\
ISQ 3 & $74.92 \pm 4.06$ & $59.92 \pm 5.97$ & - & - & - \\
$\Delta 21$ & $18.08 \pm 5.82^{\mathrm{A}}$ & $9.50 \pm 4.12^{\mathrm{B}}$ & $0.12 \pm 1.45^{\mathrm{E}}$ & $5.54 \pm 6.30^{\mathrm{D}}$ & - \\
$\Delta 31$ & $24.16 \pm 5.01^{\mathrm{A}}$ & $17.58 \pm 4.89^{\mathrm{B}}$ & - & - & - \\
\hline
\end{tabular}

For $\Delta$ variables, mean ISQ values with different letters in superscript were statistically significant (Mann-Whitney $U$ test). ISQ 1 (before grafting), ISQ 2 (after grafting), ISQ 3 (72 hours after grafting), $\Delta 21$ (increase in ISQ immediately after grafting), and $\Delta 31$ (increase in ISQ 72 hours after grafting).

${ }^{\mathrm{t}}$ Kruskal-Wallis $H$ test.

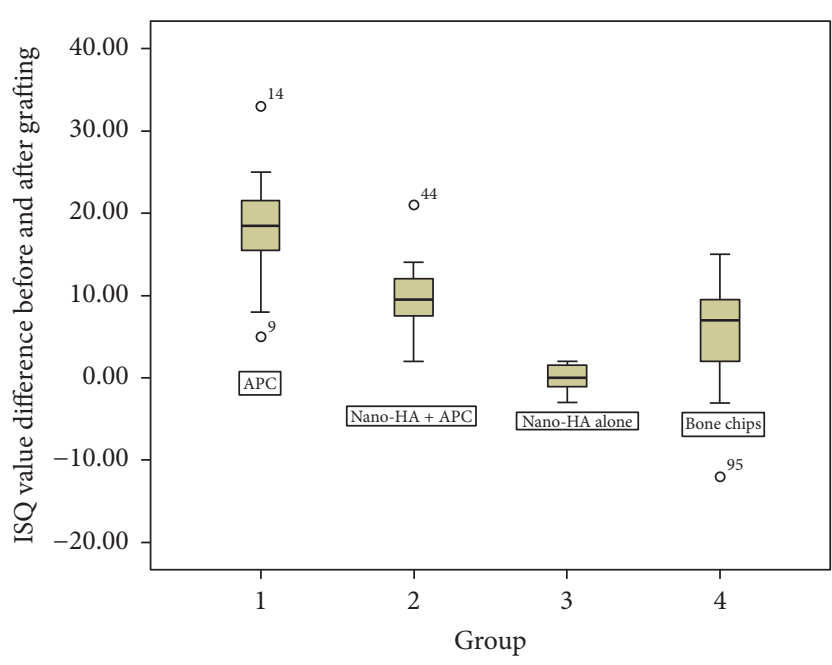

FIGURE 4: Primary stability improvements of dental implants after grafting the circumferential defects by different materials.

obtained by Poly bone $[6,20]$ and Dental Implant Bone Cement [21].

The aim of the study was to evaluate the effect of selected materials on implant initial stability However, the measurement is performed on the third day for silicate-based materials. APC generally exhibited the highest ISQ values. Although results of ISQ value improvement in APC group statistically were better than HA_APC group, but from the clinically point of view both results are acceptable and in another word the result is the same. ISQ scale ranges from 1 to 100 , but the acceptable stability range lies between 55 and 85 ISQ. The ISQ 3 (72 hours after grafting) value results for APC-Nano-HA were in acceptable range. But the NanoHA alone could not obtain acceptable ISQ value in this study. The proposed combination of APC and Nano-HA was able to make an acceptable primary stability in circumferential bone defect around dental implant (Figure 5). In our preliminary experiment, the compressive strength of Nano-HA-APC was higher than APC (1.18 versus $1.27 \mathrm{MPa})$ but, in primary stability test, ISQ value of APC was higher than NanoHA-APC group. Accordingly the compressive strength of the material cannot necessarily predict the improvement in ISQ values as well as the primary stability of implant in circumferential bone defects.

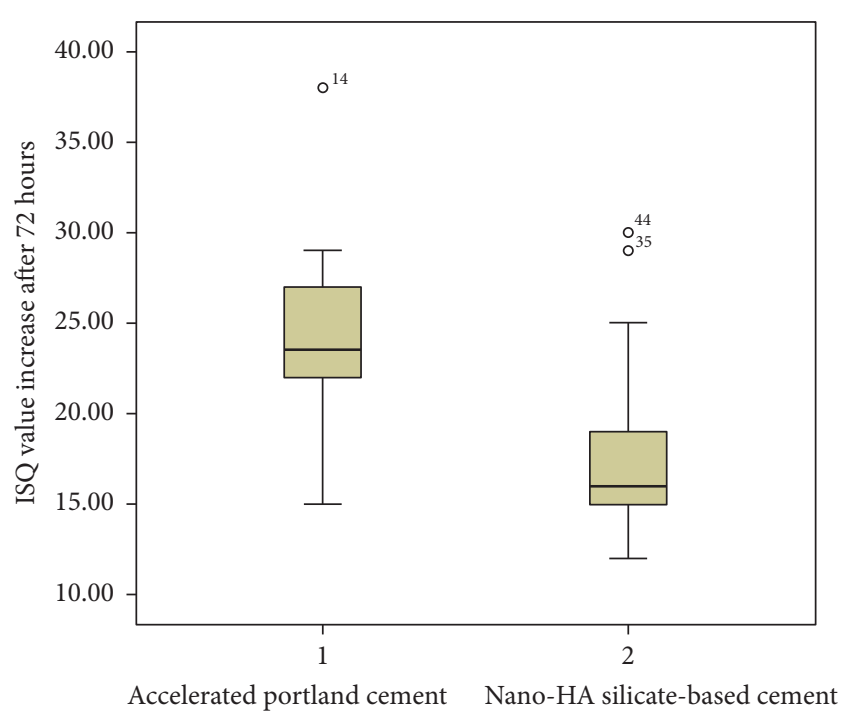

FIGURE 5: Improvement of primary stability by grafting of bone defect after 72 hours.

\section{Conclusions}

Nanohydroxyapatite powder alone could not enhance the primary stability of dental implants in the circumferential bone defects.

Silicate-based cement alone may well improve the primary stability of dental implants in the circumferential bone defects to an ISQ value, similar to an ISQ value of direct contact of implant to bone preparation sites.

Nanohydroxyapatite silicate-based cement 9 Nano-HA $75 \%$ vol and APC 25\% vol could improve the primary stability of dental implants in circumferential bone defect around implants to an acceptable stability range of ISQ value.

\section{Competing Interests}

The authors declare that there is no conflict of interests regarding the publication of this paper.

\section{Acknowledgments}

The authors appreciate the funding and support for this study provided by the Vice Chancellor of Research Center \& Innovation, Shiraz University of Medical Sciences. The 
authors wish to thank Dr. Mehrdad Vosoughi of the Dental School of Shiraz University of Medical Sciences for assistance with the statistical analysis.

\section{References}

[1] M. Ketabi, D. Deporter, and E. G. Atenafu, "A systematic review of outcomes following immediate molar implant placement based on recently published studies," Clinical Implant Dentistry and Related Research, vol. 18, no. 6, pp. 1084-1094, 2016.

[2] F. Javed and G. E. Romanos, "The role of primary stability for successful immediate loading of dental implants. A literature review," Journal of Dentistry, vol. 38, no. 8, pp. 612-620, 2010.

[3] R. Wang, S. J. Eppell, C. Nguyen, and N. Morris, "Relative contribution of trabecular and cortical bone to primary implant stability: an in vitro model study," Journal of Oral Implantology, vol. 42, no. 2, pp. 145-152, 2016.

[4] S. A. Gehrke, J. L. C. Guirado, R. Bettach, M. D. Fabbro, C. P.-A. Martínez, and J. A. Shibli, "Evaluation of the insertion torque, implant stability quotient and drilled hole quality for different drill design: an in vitro investigation," Clinical Oral Implants Research, 2016.

[5] S. A. Gandhi, J. A. Baker, L. Bairam, H.-I. Kim, E. L. Davis, and S. Andreana, "Primary stability comparison using piezoelectric or conventional implant site preparation systems in cancellous bone: a pilot study," Implant Dentistry, vol. 23, no. 1, pp. 79-84, 2014.

[6] S.-Y. Shin, S.-I. Shin, S.-B. Kye et al., "Bone cement grafting increases implant primary stability in circumferential cortical bone defects," Journal of Periodontal \& Implant Science, vol. 45, no. 1, pp. 30-35, 2015.

[7] N. F. Oliscovicz, A. C. Shimano, É. Jr. Marcantonio, C. P. Lepri, and A. C. Dos Reis, "Analysis of primary stability of dental implants inserted in different substrates using the pullout test and insertion torque," International Journal of Dentistry, vol. 2013, Article ID 194987, 5 pages, 2013.

[8] C. Prati and M. G. Gandolfi, "Calcium silicate bioactive cements: biological perspectives and clinical applications," Dental Materials, vol. 31, no. 4, pp. 351-370, 2015.

[9] S. Dokami, S. Raoofi, M. J. Ashraf, and H. Khorshidi, "Histological analysis of the effect of accelerated portland cement as a bone graft substitute on experimentally-created three-walled intrabony defects in dogs," Journal of Dental Research, Dental Clinics, Dental Prospects, vol. 1, no. 3, pp. 131-135, 2007.

[10] B. W. Darvell and R. C. T. Wu, "MTA—an hydraulic silicate cement: review update and setting reaction," Dental Materials, vol. 27, no. 5, pp. 407-422, 2011.

[11] M. G. Gandolfi, G. Ciapetti, P. Taddei et al., "Apatite formation on bioactive calcium-silicate cements for dentistry affects surface topography and human marrow stromal cells proliferation," Dental Materials, vol. 26, no. 10, pp. 974-992, 2010.

[12] H. Khorshidi, S. Raoofi, S. Sabagh, Z. Behboud, G. Mozafari, and M. J. Ashraf, "Effect of combined calcium hydroxide and accelerated portland cement on bone formation and soft tissue healing in dog bone lesions," Journal of Dental Biomaterials, vol. 2, no. 3, pp. 97-102, 2015.

[13] S. R. Dutta, D. Passi, P. Singh, and A. Bhuibhar, "Ceramic and non-ceramic hydroxyapatite as a bone graft material: a brief review," Irish Journal of Medical Science, vol. 184, no. 1, pp. 101106, 2015.
[14] H. Zhou and J. Lee, "Nanoscale hydroxyapatite particles for bone tissue engineering," Acta Biomaterialia, vol. 7, no. 7, pp. 2769-2781, 2011.

[15] J. Venkatesan and S.-K. Kim, "Nano-hydroxyapatite composite biomaterials for bone tissue engineering-a review," Journal of Biomedical Nanotechnology, vol. 10, no. 10, pp. 3124-3140, 2014.

[16] E. Pepla, "Nano-hydroxyapatite and its applications in preventive, restorative and regenerative dentistry: a review of literature," Annali di Stomatologia, vol. 5, no. 3, pp. 108-114, 2014.

[17] M. Zhao, H. Li, X. Liu et al., "Response of human osteoblast to n-HA/PEEK-quantitative proteomic study of bio-effects of nano-hydroxyapatite composite," Scientific Reports, vol. 6, Article ID 22832, 2016.

[18] S. M. Zakaria, S. H. S. Zein, M. R. Othman, F. Yang, and J. A. Jansen, "Nanophase hydroxyapatite as a biomaterial in advanced hard tissue engineering: a review," Tissue Engineering-Part B: Reviews, vol. 19, no. 5, pp. 431-441, 2013.

[19] X. Wang, H. Xing, G. Zhang et al., "Restoration of a critical mandibular bone defect using human alveolar bone-derived stem cells and porous nano-HA/collagen/PLA scaffold," Stem Cells International, vol. 2016, Article ID 8741641, 13 pages, 2016.

[20] B. M. Sehlke, T. G. Wilson, A. A. Jones, M. Yamashita, and D. L. Cochran, "The use of a magnesium-based bone cement to secure immediate dental implants," The International journal of oral \& maxillofacial implants, vol. 28, no. 6, pp. e357-e367, 2013.

[21] W.-J. Seong, S. Grami, S. C. Jeong, H. J. Conrad, and J. S. Hodges, "Comparison of push-in versus pull-out tests on bone-implant interfaces of rabbit tibia dental implant healing model," Clinical Implant Dentistry and Related Research, vol. 15, no. 3, pp. 460469, 2013. 

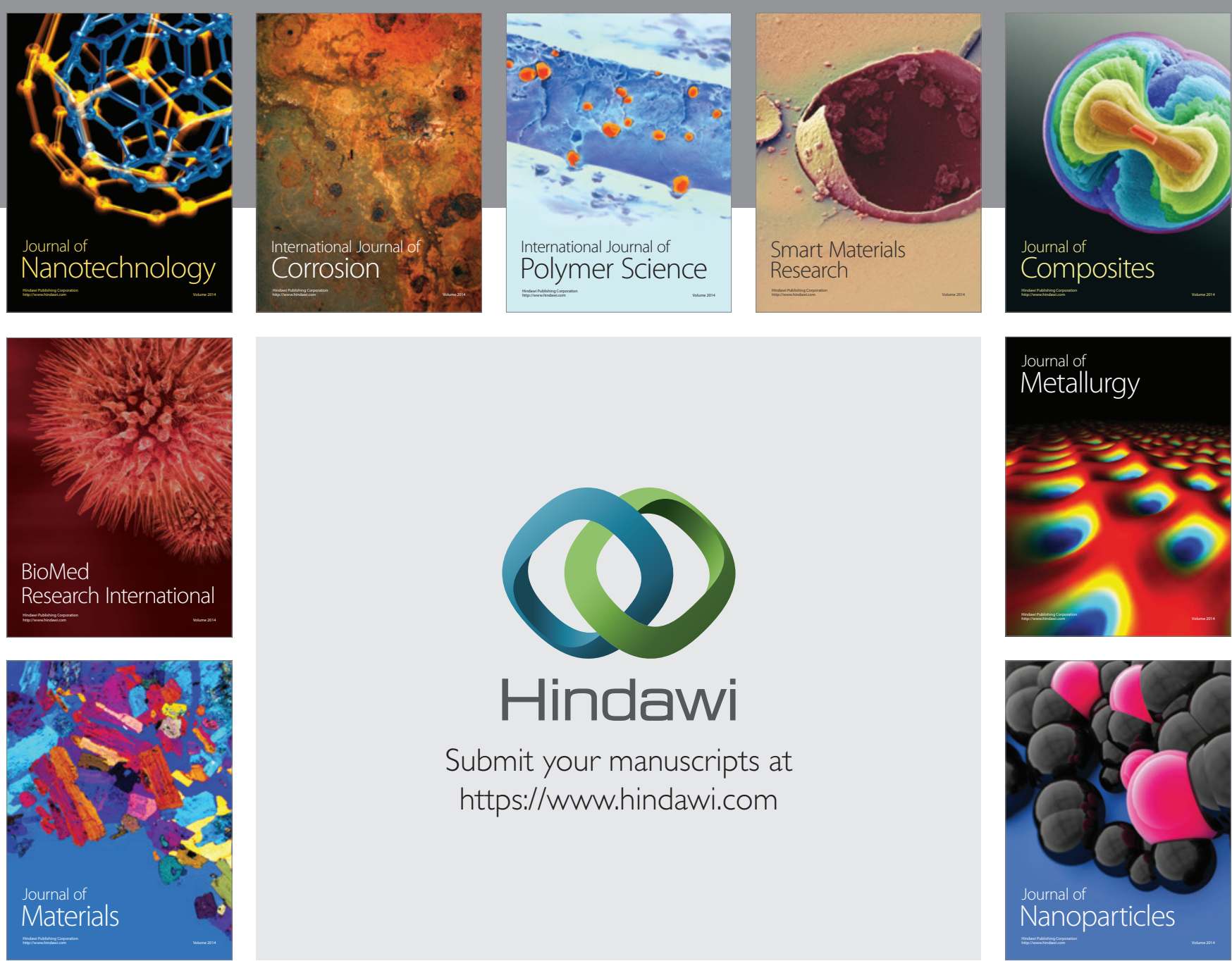

\section{Hindawi}

Submit your manuscripts at

https://www.hindawi.com

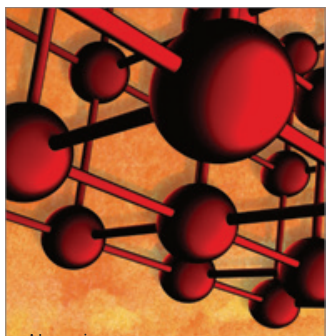

Materials Science and Engineering
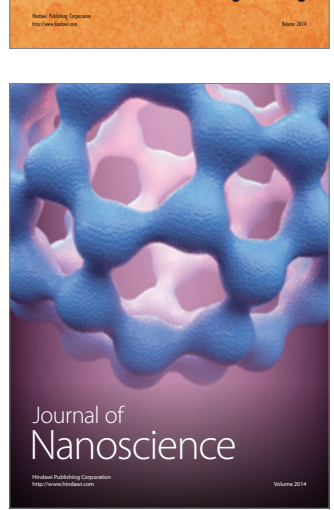
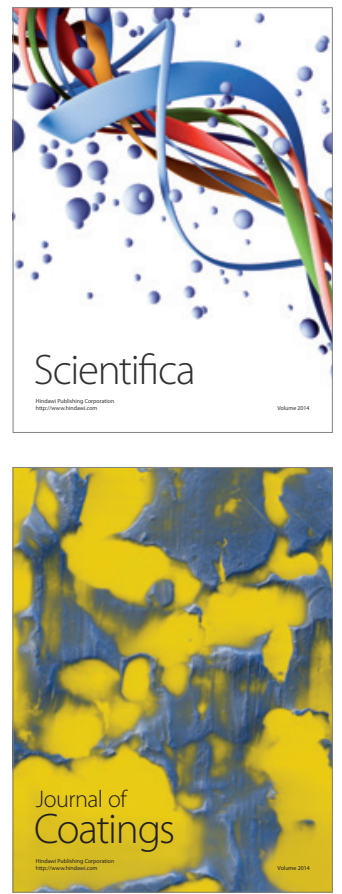
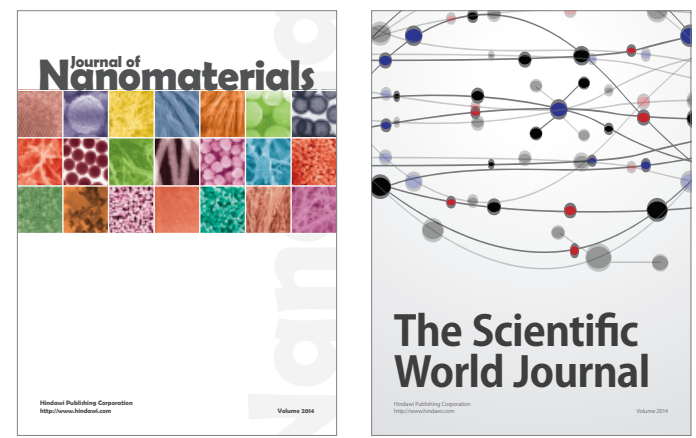

The Scientific World Journal
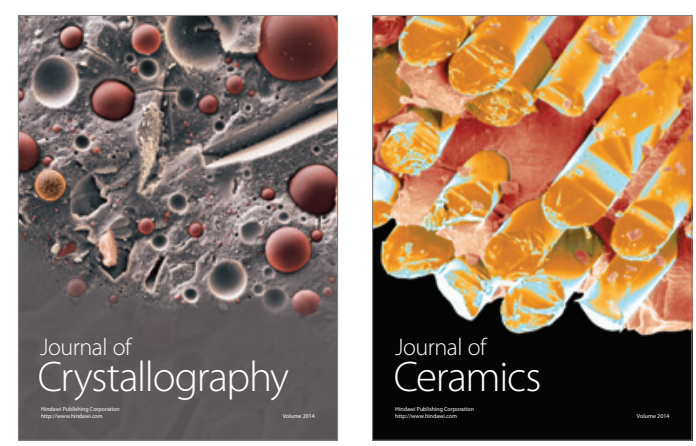
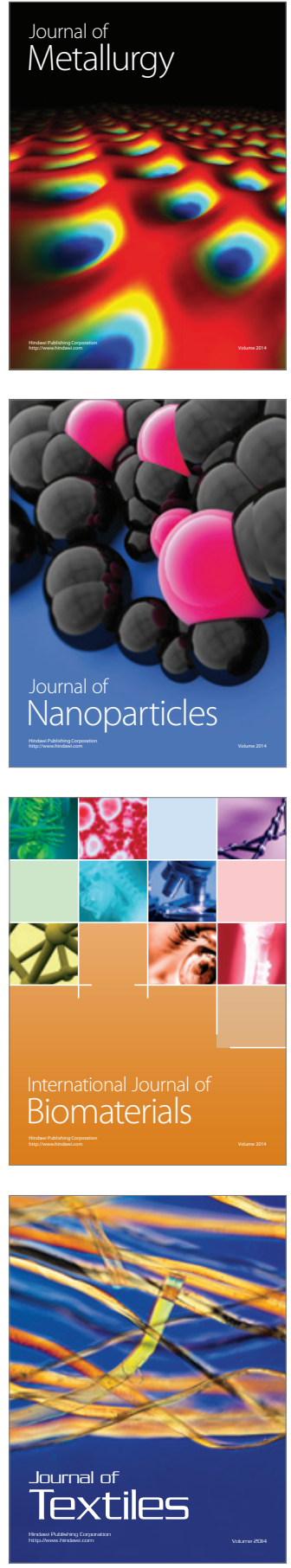